\title{
Plastic surgery in a trauma center: a multidisciplinary approach for polytrauma patients
}

\author{
Kyung-Chul Moon, MD ${ }^{\mathbb{D}}$, Yu-Kyeong Yun, MD ${ }^{\mathbb{D}}$ \\ Department of Plastic Surgery, Korea University Guro Hospital, Seoul, Korea
}

Received: October 26, 2021

Revised: November 21, 2021

Accepted: November 29, 2021

Correspondence to

Kyung-Chul Moon, MD

Department of Plastic Surgery, Korea

University Guro Hospital, 148

Gurodong-ro, Guro-gu, Seoul 08308,

Korea

Tel: +82-2-2626-1198

E-mail: boyhaha2000@daum.net
Purpose: With the increasing number of polytrauma patients treated at high-level trauma centers, plastic surgery has entered the specialty of traumatology. Plastic surgeons specialize in the simultaneous surgical care of patients with facial or hand trauma and soft tissue injuries requiring microsurgery. The purpose of this study was to introduce the role of plastic surgery in a high-level trauma center.

Methods: Between January 2020 and December 2020, 5,712 patients with traumatic injuries were admitted to the emergency department of a tertiary hospital. Of these 5,712 patients, 1,578 patients were hospitalized for surgical treatment and/or critical care. Among the 1,578 hospitalized trauma patients, 551 patients (35\%) required at least one plastic surgery procedure. The patient variables included age, sex, etiology, the injured area, and injury characteristics. We also retrospectively investigated surgical data such as the duration of the operation, hospital stay, length of time from injury to surgery, and collaboration with other departments.

Results: The most common injury referred to plastic surgery was facial trauma (41\%), followed by hand trauma (36\%), and soft tissue injuries requiring microsurgery in various parts of the body other than the hand (7\%). The majority of facial and hand traumas were concomitant injuries. Sixteen percent of patients underwent collaborative surgical management for polytrauma involving both plastic surgery and another department.

Conclusions: The role of plastic surgery in multidisciplinary teams at high-level trauma centers has become increasingly important. The results of this study may help in the development of multidisciplinary trauma team strategies and future workforce planning.

Keywords: Emergencies; Plastic surgery; Wounds and injuries; Trauma centers; Traumatology

\section{INTRODUCTION}

Trauma is the most frequent cause of death in people under 40 years of age worldwide, and trauma-related mortality is projected to increase over the next 20 years [1]. A trauma center is a hospi- tal equipped and staffed to provide care for patients with traumatic injuries from falls, motor vehicle collisions, and other events. In the United States, a hospital can receive trauma center status by meeting specific criteria established by the American College of Surgeons and passing a site review by the Verification

(C) 2022 The Korean Society of Traumatology

This is an Open Access article distributed under the terms of the Creative Commons Attribution Non-Commercial License (http://creativecommons.org/licenses/by-nc/4.0/) which permits unrestricted non-commercial use, distribution, and reproduction in any medium, provided the original work is properly cited. 
Review Committee. Trauma center designation is a process outlined and promulgated at the state or local level. The official designation as a trauma center is determined by individual state law provisions. Trauma centers vary in their specific capabilities and are identified by "level" designations, where level I is the highest and level $\mathrm{V}$ is the lowest [2].

The key elements in level I trauma centers include 24-hour inhouse coverage by general surgeons and the prompt availability of care in various specialties such as emergency medicine, internal medicine, trauma surgery, orthopedic surgery, cardiothoracic surgery, neurosurgery, anesthesiology, and critical care, as well as plastic surgery. Following the introduction of the major trauma network in Korea, 17 regional trauma centers (RTCs) and 39 regional emergency medical centers (REMCs) were designated and mandated to provide equivalent services to a highly specialized subgroup of severely injured patients.

As the number of polytrauma patients increases, plastic surgery has entered the specialty of traumatology. Plastic surgeons specialize in the simultaneous surgical care of patients with oral and craniomaxillofacial trauma, hand trauma, stab wounds, complicated wounds, deep wounds, burns, and traumatic avulsion or crushing injury requiring microsurgery. Nine years after the major trauma network was launched, plastic surgery was present at 16 of the 17 RTCs and 37 of the 39 REMCs. Two of the 39 REMCs still do not have colocated plastic surgical departments. The lack of colocated services can introduce delays in the surgical treatment of facial and hand trauma or severe soft tissue injuries requiring reconstruction using microsurgery and potentially impact the outcomes. Thirty-seven of the 39 REMCs had at least one plastic surgeon with a primary interest in facial and hand surgery. However, plastic surgery departments covering trauma centers are not standardized, although each department has a unique set of skills.

Previous studies have reported the role of plastic surgery in trauma centers specifically and individually $[3,4]$. However, limited studies have included every field of plastic surgery practice in traumatology [5]. The present study introduces the field of plastic surgery as practiced at a high-level trauma center of a tertiary hospital, equivalent to a level I trauma center in the United States.

\section{METHODS}

This retrospective cohort study was approved by the Institutional Review Board of Korea University Guro Hospital (No. 2021GR 0552). Informed consent was waived due to the retrospective nature of this study.
Between January 2020 and December 2020, 5,712 trauma patients were admitted to the emergency room of Korea University Guro Hospital. Among the 5,712 patients, 3,869 patients who met the following exclusion criteria were excluded from this study: patients who were admitted via outpatient clinics; patients with minor trauma such as contusions, sprains, and simple lacerations that received primary repair; patients with minor wounds treated with simple dressings; and patients who were not successfully monitored. In total, 1,843 patients required hospitalization for surgery and/or hospital care. Of these 1,843 patients, $262 \mathrm{pa}-$ tients were transferred to another hospital because they had sustained minor trauma (e.g., simple fractures) that did not require surgery in a tertiary hospital or due to the lack of space in a ward or an intensive care unit. Three patients died in the emergency room due to severe traumatic injuries. Therefore, a total of 1,578 patients were analyzed in this study (Fig. 1). The number of patients with an Injury Severity Score (ISS) below 10 was 987 (63\%). The number of patients with an ISS between 10 and 15 was 453 (29\%), and the number of patients with an ISS over 15 was 138 (9\%) (Fig. 2).

The primary referring surgical service was trauma surgery. The primary referring surgical service usually represents the surgical team performing the initial evaluation in a trauma center, and each service in turn determines the need for additional specialty involvement. Among 1,578 hospitalized trauma patients, 551 patients (35\%) required at least one plastic surgery procedure. The patient variables included age, sex, etiology, the injured area, and injury characteristics (Table 1).

We also retrospectively investigated surgical data, such as the duration of the operation, hospital stay, length of time from injury to surgery, and collaboration with other surgical departments (Table 2).

\section{RESULTS}

Fig. 3 presents the summary of the workflow applied by the department of plastic surgery. After the initial assessment of injuries, a complex diagnostic procedure was completed to determine the therapeutic needs in the emergency room. A treatment plan was then prepared, and surgical treatment was carried out as soon as possible. In patients with prominent hemodynamic instabilities such as low blood pressure due to massive bleeding, brain hemorrhage, hemoperitoneum, hemothorax, and cardiac tamponade, plastic surgery was performed despite the presence of open injuries on the face or hand. In patients with open injuries who were hemodynamically stable, plastic surgery was per- 


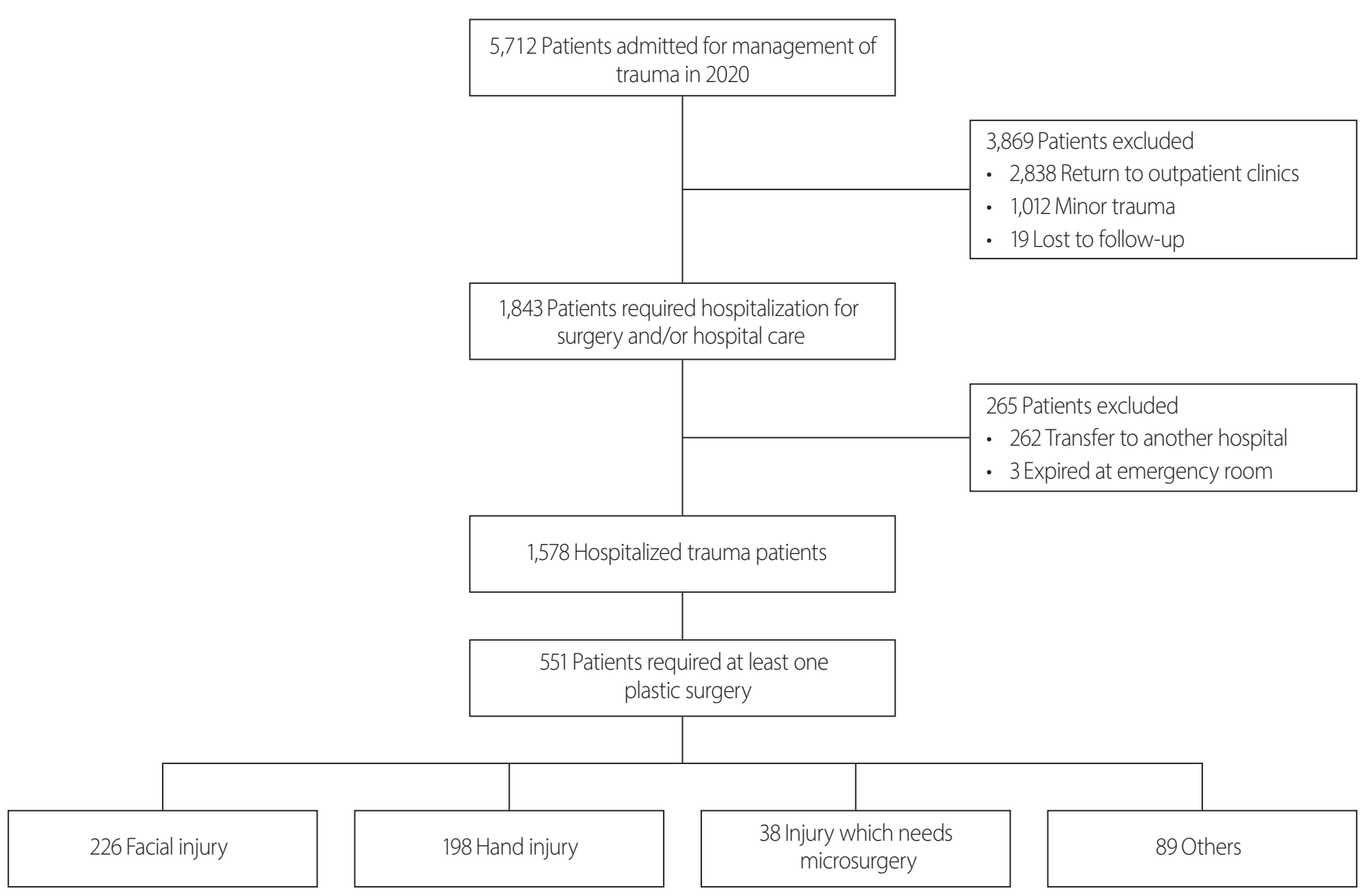

Fig. 1. Flow diagram of patients included in this study.

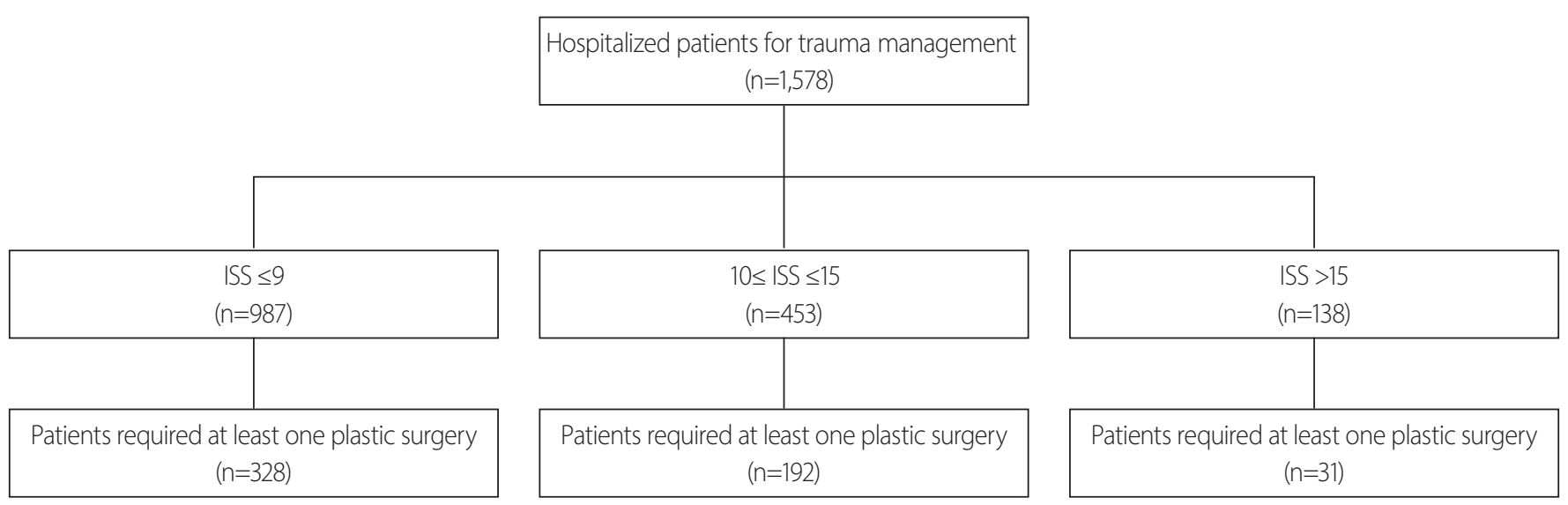

Fig. 2. Classification of patients according to the Injury Severity Score (ISS).

formed within 24 hours. In cases of replantation or an avulsion/ crushing injury with main vessel rupture that needed microsurgery, the anastomosis of vessels was performed by a plastic surgeon. Patients with closed fractures underwent surgery within 7 days.

A total of 551 patients underwent plastic surgery during the study period. Among 551 patients, 89 patients (16\%) underwent collaborative surgical management for polytrauma involving plastic surgery and another department. Orthopedic surgery accounted for the highest proportion of collaborations, followed by neurosurgery and trauma surgery. Within the plastic surgery cohort, the male to female ratio was 1.6:1, and the mean age was 
Table 1. Patient demographics

\begin{tabular}{lc}
\hline Characteristic & Value \\
\hline Total & 551 \\
Sex (male:female) & $340: 211$ \\
Age (yr) & $45.1 \pm 12.7(5-83)$ \\
Follow-up (mo) & $24.3 \pm 7.1(5-59)$ \\
Etiology & 551 \\
Vehicle accident & $314(57)$ \\
Fall from height & $94(17)$ \\
Work-associated injury & $75(14)$ \\
Assault & $38(7)$ \\
Others & $30(5)$ \\
Injured area & \\
Face & $226(41)$ \\
Hand & $198(36)$ \\
Lower extremity & $72(13)$ \\
Trunk & $11(2)$ \\
Others & $44(8)$ \\
Injury characteristics & \\
Closed fracture & $152(55)$ \\
Open fracture & $118(25)$ \\
Incomplete/complete amputation & $52(9)$ \\
Soft tissue injury without fracture & $229(42)$ \\
Deep laceration requiring surgery & $140(25)$ \\
$\quad$ Burn & $57(10)$ \\
Infected wound & $32(6)$ \\
\hline
\end{tabular}

Values are presented as number, mean \pm standard deviation, or number (\%).
Table 2. Surgical details

\begin{tabular}{lc}
\hline Characteristic & Value \\
\hline Plastic surgery & 551 \\
Facial surgery & $226(100)$ \\
Nasal bone fracture & $52(23)$ \\
Blow-out fracture & $36(16)$ \\
Zygomatic fracture & $31(14)$ \\
Mandible fracture & $17(8)$ \\
Frontal sinus fracture & $10(4)$ \\
Concomitant fractures & $62(27)$ \\
Soft tissue injury without fracture & $18(8)$ \\
Hand surgery & $198(100)$ \\
Fracture & $44(22)$ \\
Tendon surgery & $42(21)$ \\
Vessel surgery & $28(14)$ \\
Nerve surgery & $14(7)$ \\
Concomitant surgery including replantation & $70(35)$ \\
Microsurgery except for hand & 38 \\
Others (e.g., deep laceration requiring exploration, & 89 \\
burn, complicated wound) & $1.2 \pm 0.4$ \\
Operation time (hr) & $6.3 \pm 5.3$ \\
Hospital stay (day) & $2.3 \pm 3.1$ \\
Time from injury to surgery (day) & $89(100)$ \\
Collaboration with other surgery departments & $21(24)$ \\
Orthopedic surgery & $10(11)$ \\
Neurosurgery & $6(7)$ \\
Trauma surgery & $52(58)$ \\
More than two surgery departments & \\
\hline &
\end{tabular}

Values are presented as number, number $(\%)$, or mean \pm standard deviation.

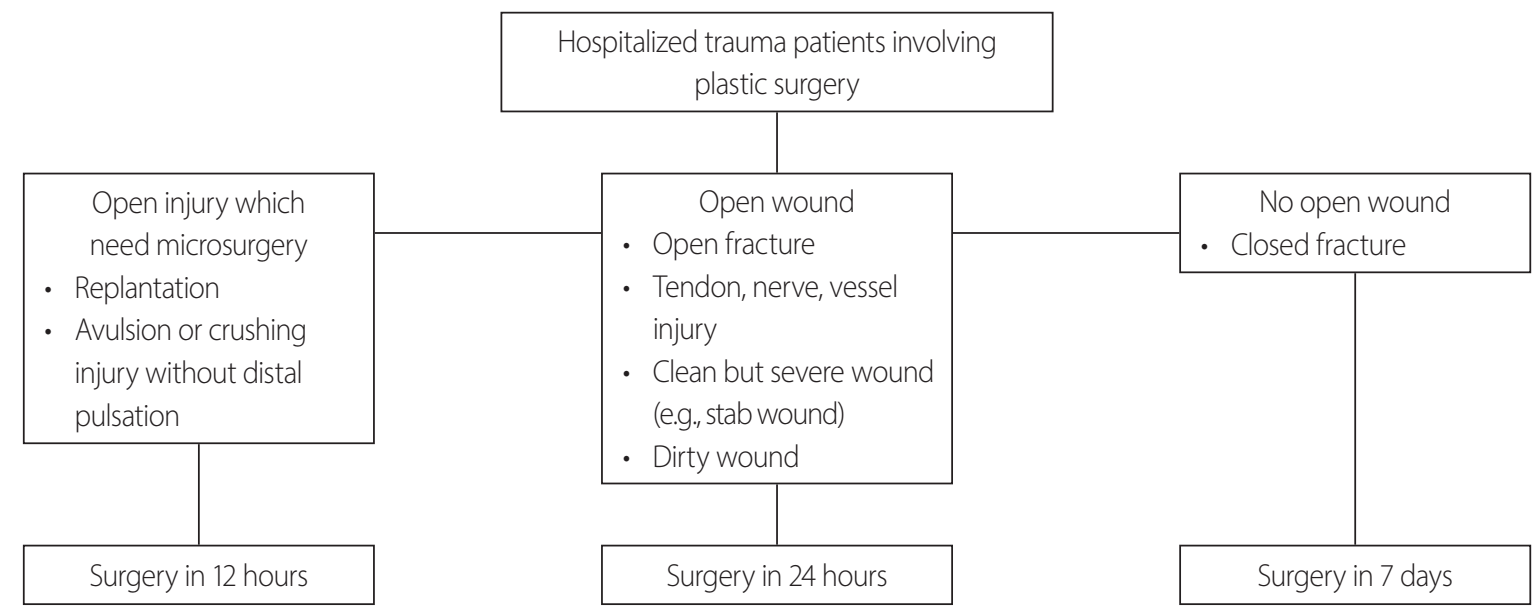

Fig. 3. Summary of the workflow applied by plastic surgery. 
45.1 years (range, 5-83 years). The most frequent mechanisms of injury were vehicle accidents (314 patients, $57 \%$ ), followed by falls from a height (94 patients, 17\%), and work-related trauma (75 patients, 14\%). The mean ISS of the plastic surgery patients was 8.4.

Facial trauma was the most common anatomical area operated on, and hand trauma surgery represented the second most frequent anatomical area treated with plastic surgery. The majority of the facial traumas were concomitant injuries, followed by nasal bone fractures, blow-out fractures, zygomatic fractures, soft-tissue injuries without fractures, mandible fractures, and frontal sinus fractures. The majority of the hand traumas were also concomitant injuries, followed by fractures and soft-tissue injuries such as tendon, nerve, and vessel injuries. Microsurgery in an area other than the hand was needed in $7 \%$ of patients after recovery.

\section{DISCUSSION}

Trauma care is a prototypical example of a multidisciplinary team effort in which surgeons provide leadership. Trauma surgery, neurosurgery, and orthopedic surgery have been regarded as core disciplines. The comprehensive management of critically injured patients is demanded in high-level trauma centers. However, the pivotal role of the plastic surgeon warrants recognition [3]. The role of the plastic surgeon in cosmetic procedures is well understood by the public and the medical profession. However, the role of plastic surgery in traumatology is not well known.

In traumatology, the role of plastic surgery lies in facial and hand trauma, as well as trauma requiring vessel anastomosis using microsurgery. Furthermore, the role of plastic surgery in reconstruction using skin grafts, local flaps, and free flaps is also important for severe trauma patients after recovery. This study demonstrated that the scope of plastic surgery amenable to care by plastic surgeons extended well beyond these boundaries. To a large extent, this is afforded by the training that occurs in both soft-tissue surgery and osteosynthesis in programs with strong plastic and reconstructive foundations. The tremendous flexibility of the specialty afforded by this training allows for overlap and interactions with a number of other surgical specialties. This overlap can be capitalized on both by working in conjunction with other surgical services and by using plastic surgeons to cross-cover for other surgical specialties where a surgeon shortage exists [3].

Traumatic injuries significantly affect patients socially, functionally, and financially. It is estimated that the work-loss costs for the survivors of traumatic injuries amount to $\$ 167$ billion annually in the United States alone [6]. There is growing evidence that the victims of traumatic injuries experience reduced quality of life, poor psychosocial outcomes, limitations in the activities of daily living, chronic pain, and psychological disturbances $[7,8]$. In particular, patients with facial injuries represent a subset of the entire trauma population that may be at increased risk for worse long-term outcomes due to the nature of their injuries [9]. The face plays an important role in human interactions and in communicating intentions and emotions, and it is closely tied to our sense of identity and self-worth $[9,10]$. Injuries within the scope of plastic surgery are usually not life-threatening [4]. However, injuries related to plastic surgery may result in considerable disabilities and diminished quality of life [4]. In addition, functional recovery following severe hand trauma is essential in daily life.

This study was a retrospective assessment of the annual treatment in 5,712 trauma patients at a tertiary hospital. In this study, plastic surgery operative input was required in at least 35\% of major trauma patients undergoing hospitalization. In recent years, multidisciplinary team efforts involving trauma surgery, orthopedic surgery, neurosurgery, cardiac surgery, plastic surgery, and critical care teams have provided leadership in this high-level trauma center in southwest Seoul. The trauma coordinators have meetings every week and discuss all patients with an ISS of more than 9, as well as polytrauma patients.

The Korea University Medical Center is a Ministry of Health and Welfare-verified regional trauma medical center with a pediatric commitment. It serves as a key resource facility in a regional trauma system implemented in 2012. Until 1983, Korea University Guro Hospital provided severe trauma coverage on a contractual basis. Guro Industrial Corporation was located near Korea University Guro Hospital and many severe trauma patients from its factories were referred to Korea University Guro Hospital. Historically, the first full-time plastic surgeon was assigned to Korea University Guro Hospital in 1983, and more than 500 finger replantation and thousands of hand traumas were surgically managed, particularly by plastic surgeons.

Since polytrauma management requires knowledge of the complex spectrum of medical management as well as surgical treatment, it necessitates collaboration and partnership between multiple departments. The collaboration between plastic surgery and other departments facilitates the elaboration of comprehensive therapeutic plans and helps to determine the timing of surgery and the appropriate management of severe trauma patients. In patients with severe trauma due to falls from a height and vehicle accidents, complex traumatic lesions are frequent and a 
multidisciplinary approach to treat polytrauma patients is needed. To enhance the specialization of a high-level trauma center, identification of even minor trauma and the proper management thereof are essential.

The orthopedic-plastic partnership also led to a reduction of $67 \%$ in the number of surgical debridements and decreased hospital stays by $79 \%$, which represented a major economic advantage [11]. A multicenter prospective study including 545 patients was conducted to increase the quality of life and decrease the costs of complex lower limb trauma management [12]. Another multicenter study reported a statistically significantly higher risk of infections in cases with solely orthopedic treatment of open fractures. In contrast, the consolidation duration was significantly shorter in cases involving "ortho-plastic" collaboration [13]. Surgical debridement, bone fixation, and flap coverage may be required in severe trauma patients with open fractures of the upper and lower extremities. After bone fixation by orthopedic surgeons, appropriate wound management and flap coverage may be performed by plastic surgeons. In this context, the plastic surgeon is no longer merely an occasional actor dedicated to cutaneous covering alone, but a key team member participating from admission onwards in the elaboration of therapeutic strategies. Thus, the creation of networks for the rapid treatment of patients with complex injuries in specialized trauma centers is of paramount importance for severe trauma patients after recovery.

This study clearly defined the need for trauma and plastic surgeons to operate in major trauma centers. The unique capability of plastic surgeons lies in providing resuscitative surgical techniques while also maintaining long-term perspectives on plastic and reconstructive surgery. In addition, their capabilities will undoubtedly improve patient outcomes by encouraging a conservative and pragmatic approach to trauma and wound management [1]. The authors suggest that each plastic surgery department serving in an REMC should include surgeons with skills relevant to the types of injuries presented, including head and neck surgeons, extremity trauma surgeons, and access to reconstructive surgeons. Because of the necessarily multidisciplinary nature of most major trauma work, plastic surgeons supporting major trauma centers should also be familiar and comfortable with working closely with related surgical and medical specialties, as well as allied healthcare professionals [5].

The primary limitation of this study is its retrospective nature. Another limitation of this study is that it was a monocentric study. A multicenter, prospective extension of this study would bring more scientific value. However, this would require continuous efforts at all research centers, which can increase long-term bias due to nonreported cases. In addition, patients admitted via outpatient clinics were not included in this study. Therefore, the number of trauma patients may have been higher, even though there was no difference in the ratio of plastic surgery patients to total trauma patients.

Despite these limitations, we performed this epidemiological study to investigate the specialty of plastic surgery and its role in the multidisciplinary team of a high-level trauma center. These results can be helpful in establishment of treatment strategies in polytrauma patients involving plastic surgery because plastic surgery patients often need collaboration with other surgical departments. Although this epidemiological study of plastic surgery in traumatology is not comprehensive enough to ensure the prevention of trauma, this study specifically analyzed the role and treatment plan of plastic surgery among trauma physicians belonging to various departments. Although a further multicenter largescale study is required, this study may provide useful information for physicians engaged in the assessment of plastic surgery patients.

In conclusion, plastic surgery may make a substantial contribution to major trauma surgical treatment, and the majority of this workload was found to be related to facial and hand trauma and injuries requiring microsurgery in a high-level trauma center. Recognition of the volume and nature of plastic surgery in a trauma center is vital for ensuring the provision of an adequate and relevant workforce and guiding service designs to achieve the best outcomes for high-level trauma centers. The diagnostic and treatment protocols of plastic surgery in polytrauma patients should be appropriate. Both these factors and sufficient experience support good results and low complication rates. A multidisciplinary approach for polytrauma patients may reduce the complication rate, and the current guidelines and workflow may contribute to improving patient safety and treatment outcomes.

\section{NOTES}

\section{Ethical statements}

This retrospective cohort study was approved by the Institutional Review Board of the Korea University Guro Hospital (No. 2021GR0552). Informed consent was waived due to the retrospective nature of this study.

\section{Conflicts of interest}

The authors have no conflicts of interest to declare. 


\section{Funding}

This research was supported by the Basic Science Research Program through the National Research Foundation of Korea (NRF) funded by the Ministry of Education and Korea University Guro Hospital (No. 2021M3E-5D1A01015189).

\section{Author contributions}

Conceptualization: KCM; Data curation: all authors; Formal analysis: all authors; Funding acquisition: KCM; Methodology: KCM; Project administration: KCM; Visualization: all authors; Writing-original draft: KCM; Writing-review \& editing: KCM. All authors read and approved the final manuscript.

\section{REFERENCES}

1. Staruch RM, Lloyd-Hughes H, Shiatis A, Hettiaratchy SP. Evidence for the formal development of trauma subspecialty within plastic surgery in the United Kingdom. J Plast Reconstr Aesthet Surg 2018;71:e8-9.

2. Brown JB, Watson GA, Forsythe RM, et al. American College of Surgeons trauma center verification versus state designation: are level II centers slipping through the cracks. J Trauma Acute Care Surg 2013;75:44-9.

3. Peterson SL, Moore EE. The integral role of the plastic surgeon at a level I trauma center. Plast Reconstr Surg 2003;112: 1371-5.

4. Hacikerim Karsidag S, Ozkaya O, Ugurlu K, Bas L. The practice of plastic surgery in emergency trauma surgery: a retrospective glance at 10,732 patients. Ulus Travma Acil Cerrahi Derg 2011;17:33-40.

5. Hendrickson SA, Young K, Gardiner MD, et al. The role of plastic surgery in major trauma in the United Kingdom and workforce recommendations. J Plast Reconstr Aesthet Surg 2021;74:1071-6.

6. Florence C, Haegerich T, Simon T, Zhou C, Luo F. Estimated lifetime medical and work-loss costs of emergency department-treated nonfatal injuries: United States, 2013. MMWR Morb Mortal Wkly Rep 2015;64:1078-82.

7. Herrera-Escobar JP, Seshadri AJ, Stanek E, et al. Mental health burden after injury: it's about more than just posttraumatic stress disorder. Ann Surg 2021;274:e1162-9.

8. Haider AH, Herrera-Escobar JP, Al Rafai SS, et al. Factors associated with long-term outcomes after injury: results of the Functional Outcomes and Recovery after Trauma Emergencies (FORTE) multicenter cohort study. Ann Surg 2020;271: 1165-73.

9. McCarty JC, Herrera-Escobar JP, Gadkaree SK, et al. Longterm functional outcomes of trauma patients with facial injuries. J Craniofac Surg 2021;32:2584-7.

10. Rumsey N, Harcourt D. Body image and disfigurement: issues and interventions. Body Image 2004;1:83-97.

11. Sargazi N, El-Gawad A, Narayan B, et al. A full time regional ortho-plastic unit; initial results. J Plast Reconstr Aesthet Surg 2016;69:572-3.

12. MacKenzie EJ, Bosse MJ, Pollak AN, et al. Long-term persistence of disability following severe lower-limb trauma. Results of a seven-year follow-up. J Bone Joint Surg Am 2005; 87:1801-9.

13. Boriani F, U1 Haq A, Baldini T, et al. Orthoplastic surgical collaboration is required to optimise the treatment of severe limb injuries: a multi-centre, prospective cohort study. J Plast Reconstr Aesthet Surg 2017;70:715-22. 